\title{
CONSTRUCCIÓN SOCIAL DEL RIESGO EN ZONAS RURALES DE LA COMUNA DE PUTRE, CHILE
}

Daniela Aragón Urtubia ${ }^{1, *}$

\section{RESUMEN}

El presente estudio describe la construcción social del riesgo desde la percepción de los habitantes de Putre, quienes conviven con amenazas hidrometeorológicas como lluvias estivales. Estas últimas reconocidas como lluvias altiplánicas. Para su desarrollo se utilizó una metodología cualitativa, aplicando entrevistas en profundidad. Instrumento que permitió conocer las capacidades y vulnerabilidades identificadas por personas que habitan áreas rurales, cuyos datos fueron analizados mediante el Análisis de Vulnerabilidades y Capacidades. Para lo anterior, se trabajó desde el modelo de Presión y Liberación del riesgo, que comprende la vulnerabilidad como potenciador del riesgo, exponiendo a comunidades ante desastres. Desde esta mirada, se percibe al riesgo como construcción social entre amenaza y vulnerabilidad, pudiendo mitigarse a partir del fortalecimiento de capacidades individuales y comunitarias. En resultados, se destacó el análisis de recursos sociales, resiliencia y participación. En el área rural, indicó que durante post emergencia se despliegan prácticas de preparación ante lluvias. Por el contrario, el área urbana expresa que la presencia de instituciones y de población de avanzada edad, son factores de la perdida de acciones preventivas.

\section{PALABRAS CLAVE}

Riesgo, Percepción, Capacidades, Vulnerabilidad, Gestión

\section{SOCIAL CONSTRUCTION OF RISK IN RURAL AREAS OF PUTRE, CHILE}

\section{ABSTRACT}

The study describes the social construction of risks from the perception of the residents of Putre, in Chile, who coexist with hydro-meteorological hazards such as heavy rains during summer, also named 'altiplanic' rains. Based on a qualitative methodology, the research uses in-depth interviews to describe the capacities and vulnerabilities of people living in rural areas. Data were collected and analyzed through the Vulnerabilities and Capacities Analysis method, and complemented through the Pressure and Release model. The latter allowed to consider vulnerability as a risk enhancer and determinant for community exposure to disasters. From this perspective, risk was perceived as a social construction mediated by the interaction of vulnerability and hazard, while it can be mitigated by strengthening individual and community capacities. Results highlight the analysis of social resources, resilience and participation. Rural areas tend to deploy preparedness measures during post-disaster periods, while urban areas tend to believe that the presence of institutions and a high number of elderly people are factors of the loss of preventive actions.
\end{abstract}

\section{KEYWORDS}

Risk, Perception, Capabilities, Vulnerability, Management
1. Fundación Superación de la Pobreza, Dirección Regional Arica y Parinacota \& Programa SERVICIO PAÍS Arica, Región de Arica y Parinacota, Chile.

*Autor de correspondencia: danielaaragon83@gmail.com

\section{RECIBIDO}

7 de mayo de 2018

\section{ACEPTADO}

18 de junio de 2018

\section{PUBLICADO}

25 de julio de 2018

\section{Formato cita}

Recomendada (APA): Aragón Urtubia, D. (2018). Construcción social del riesgo en zonas rurales de la comuna de Putre, Chile. Revista de Estudios Latinoamericanos sobre Reducción del Riesgo de Desastres REDER, 2(2), pp.61-78

\section{(ल) $(\Phi \otimes$}

Todos los artículos publicados en REDER siguen una política de Acceso Abierto y se respaldan en una Licencia CreativeCommons Atribución-NoComercial 4.0 Internacional.

\section{Revista de Estudios} Latinoamericanos sobre Reducción del Riesgo de Desastres (REDER)

Diseño: Lupe Bezzina Tipografía: Hospital 


\section{INTRODUCCIÓN}

La construcción social del riesgo revalora capacidades e identifica vulnerabilidades relacionadas a amenazas que impactan continuamente los medios de vida. Este enfoque permite conocer estrategias desplegadas por los habitantes de Putre, quienes cada año se ven enfrentados a riesgos producto de lluvias estivales. Por lo anterior cabe preguntarse cuáles son las capacidades y vulnerabilidades que identifican los miembros del comité de protección civil y dirigentes vecinales, ante posibles desastres hidrometeorológicos. A fin de dar respuesta a la investigación, se releva la construcción social del riesgo ante desastres hidrometeorológicos, mediante la percepción de capacidades y vulnerabilidades. Para lo cual, se identificaron vulnerabilidades y reconocieron capacidades ante posibles desastres, a través de descripción de medios de subsistencia, bienestar, autoprotección, protección social y gobernanza.

Para el alcance de los objetivos, se utilizó categorías del Método de Análisis de Vulnerabilidades y Capacidades (AVC), comprendido desde el modelo de presión y liberación de riesgos. Esto permitió entender las capacidades y vulnerabilidades de los entrevistados desde la comprensión de su hábitat.

En los resultados, se visualizó una serie de prácticas comunitarias y experiencias desde la ruralidad, donde generaba resiliencia y adaptación al medio, como característica personal y comunitaria.

Este estudio pretende ser un material complementario en el trabajo de gobiernos locales, siendo parte de un enfoque multidisciplinar, sensibilizando y promocionando una cultura reductiva y preventiva ante riesgos de desastre hidrometeorológicos.

\section{CONTEXTO Y MARCO TEÓRICO}

Putre es un territorio con presencia de factores relacionados a riesgos. Aquellos están vinculados a amenazas naturales y antrópicas, por ejemplo, el fenómeno del 'Invierno Altiplánico" que produce aumento de precipitaciones, pudiendo provocar riesgos de inundación, desplazamiento, remoción de masa, entre otros (llustre Municipalidad de Putre, 2014).

En procesos participativos de la Corporación Nacional Forestal Chilena (CONAF), acerca de la Estrategia sobre Cambio Climático y Recursos Vegetacionales, personas aymaras indicaron presenciar la variabilidad climática, mediante la siguiente cita: "En pleno diciembre del año pasado, tuvimos escarchas y nos quemaron las chacras" (Arica Mía, 2016). Por este motivo se consideró que los habitantes de Putre pueden llegar a percibir los riesgos por temporadas, considerando el invierno altiplánico como principal causa de pérdidas agrícolas y ganaderas.

Asimismo, la Fundación Superación de la Pobreza (2016) indica que el recurso hídrico es un factor relevante para preservar el paisaje altiplánico y de precordillera, reconoce que la geografía y biodiversidad de la región posee un gran potencial de desarrollo turístico. Del mismo modo, las precipitaciones estivales son observadas como un elemento importante para el desarrollo.

La comprensión del riesgo de desastres es entendida en este trabajo desde la perspectiva social. Lavell (2000) indica que el 'paradigma social' o de 'vulnerabilidad ante desastres' se relaciona a que estos son causados por tipos de modelos de desarrollo. Por lo tanto, la generación del riesgo desde este enfoque, es ocasionada por fallidas planificaciones del ámbito social, económico y político.

El enfoque sobre la vulnerabilidad es complementado por el Modelo de Presión y Liberación de Desastres (PAR, por sus siglas en inglés), donde Blaikie, Cannon, Davis y Wisner (1996) proponen entender las causas de las catástrofes, como una:

"Intersección de dos fuerzas opuestas: los procesos generadores de vulnerabilidad por un lado y exposición física (amenaza) por el otro. Comprendiéndose como un cascanueces, con mayor presión en la población que surge con diversos grados de vulnerabilidad e impactada por amenazas. El concepto sobre liberación, se incorpora para explicar la reducción del desastre: por consecuencia atenuando la presión, la vulnerabilidad tiende a reducir"

(Blaikie et al., 1996, p.28)

1. Lluvias Estivales, conocido popularmente como "Invierno del Altiplano Chileno", donde se generan chubascos y precipitaciones, tempestades eléctricas, granizos, nevadas, en los meses de diciembre a marzo en la Cordillera de los Andes (Bravo Flores, 2002) 
Según lo señalado, la reducción de desastres se consigue mitigando la vulnerabilidad. Por lo tanto, el fomento de capacidades comunitarias reduce los riesgos, permitiendo minimizar la exposición a entornos vulnerables.

Complementando lo anterior, García (2005) postula que los riesgos de desastres son construidos socialmente y están asociados a percepciones, vulnerabilidad y desigualdad. Indica que "Riesgo es un conjunto de conocimientos y aceptación, dependiente de la percepción que se tenga de él. La percepción del riesgo es entonces un proceso social y una construcción cultural" (García , 2005 , p.16). Por lo tanto, la gestión y reducción de riesgos de desastres (GRRD), se entiende como una estrategia integral para el desarrollo sostenible, que busca crear comunidades resilientes mediante la promoción de sus recursos y capacidades. La Gestión del Riesgo de Desastre es comprendida por Lavell (2003), como:

"Un proceso social complejo, cuyo fin es la reducción o la previsión y control permanente del riesgo de desastre en la sociedad, integrada al logro de pautas de desarrollo humano, económico, ambiental y territorial sostenibles. Admite, en principio, distintos niveles de coordinación que van desde lo global, integral, lo sectorial y lo macro-territorial hasta lo local, lo comunitario y lo familiar".

(Lavell, 2003, p.30)

De esta manera, la GRRD se construye en relación a procesos vividos y promoviendo una cultura resiliente. Del mismo modo, las instituciones deben propiciar una participación activa de la sociedad civil y privados, estableciendo colaboraciones técnicas, tomando como base el ciclo de gestión del riesgo de desastre.

CARITAS (2015) destaca que la apropiación por parte de la comunidad es primordial en comprender el componente local de gestión del riesgo, entendiéndose como una instancia diferente a la gestión municipal. Dando a conocer que existen procesos originados a nivel local por comunidades, tornándose importante en su valoración popular y prácticas preventivas en reducción de desastres.

La gestión del riesgo de desastres o GRD, en sus esferas de acción, produce una organización de actores y recursos en fases interdependientes. En el estudio se observó a personas, desde sus capacidades y contextos vulnerables en el antes, durante y después del ciclo del riesgo, permitiendo comprender de mejor forma su organización. A continuación se profundiza en la construcción social del riesgo, sus componentes y enfoques.

La construcción social del riesgo se comprendió desde el dinamismo de los riesgos, donde vulnerabilidades y capacidades hacen que personas y comunidades den diferentes significados. Del mismo modo, Lavell (2003) señala que los riesgos se encuentran sujetos a análisis objetivos y subjetivos, donde la GRRD implica tomar decisiones en ambos aspectos.

De esta forma, García Acosta (2005) indica que el riesgo es percibido de diferentes formas por las sociedades, a pesar de tener similares amenazas. Esta variabilidad, se debe a que es el ser humano el que elige distinguir lo peligroso y no peligroso, aceptando sus implicancias. Además, agrega que "El riesgo no es un ente material objetivo, sino una elaboración, una construcción intelectual de los miembros de la sociedad, que se presta particularmente para evaluaciones sociales de probabilidades y valores" (García Acosta, 2005, p.15)

Los aspectos anteriores demuestran la importancia del rol comunitario en el reconocimiento de los peligros. Desde esta perspectiva, Thomas (2011) indica que la construcción social del riesgo debería estar implícita en las políticas de desarrollo, e indica que:

"Es fundamental reconocer el rol del Estado y la sociedad alrededor de condiciones que definen la vulnerabilidad social de poblaciones. La concepción y percepción social del riesgo y tipos de vulnerabilidad se materializa en una política pública, resulta esencial para la generación de contradicciones entre prioridades económicas y condiciones seguras de las comunidades"

(Thomas, 2011, p.137) 
Lo mencionado da cuenta que los autores reconocen el rol de las sociedades en la construcción del riesgo, donde el sentido de sus percepciones y experiencias forman parte de su habitar.

Complementando lo anterior, el AVC comprende la construcción social del riesgo mediante el análisis de capacidades y vulnerabilidades. Este enfoque recopila información territorial, diagnosticando riesgos y capacidades comunitarias (FICR, 2006). Bajo este modelo se utilizaron los siguientes criterios analíticos: medios de subsistencia, bienestar de las personas, autoprotección, protección social y gobernanza. A continuación se explica cada uno.

1. Medios de subsistencia: son activos utilizados por las personas para enfrentar los riesgos. Capacidades que al fortalecerse minimizan vulnerabilidades. La Organización de Naciones Unidas para la Alimentación y la Agricultura (FAO) (2006) explica:

"Un medio de vida se compone de capacidades, activos (tanto recursos materiales como sociales) y actividades necesarias para vivir. Un medio de vida es sostenible cuando puede afrontar y recuperarse de rupturas y caídas bruscas, y mantener sus capacidades y activos tanto en el presente como en el futuro sin socavar las base de recursos naturales"

(FAO, 2006, párr.5)

A diferencia de la definición anterior, Kaztman y Filgueira (1999) distinguen entre recursos y activos, señalando que "Los recursos transitan a activos a medida que permiten el aprovechamiento de oportunidades ofrecidas por el Estado, mercado o sociedad" (Kaztman \& Filgueira, 1999, p.8). De esta manera, las personas y comunidades tienen diversos recursos, pudiendo ser activados mediante acceso a una estructura de oportunidades y resiliencia en situaciones adversas.

Igualmente estos autores indican que: "El nivel de vulnerabilidad de un hogar se refiere a su capacidad para controlar las fuerzas que lo afectan, depende del control de activos y de recursos requeridos para el aprovechamiento de oportunidades que brinda el medio donde se desenvuelve" (Kaztman \& Filgueira, 1999). En este sentido, personas, hogares y comunidades enfrentan mejor los riesgos estando empoderadas y vinculadas a una estructura de oportunidades.

No existe una sola categoría de activos que baste para alcanzar la sostenibilidad comunitaria. Más bien, es una diversidad que, en conjunto, permite alcanzar resiliencia y empoderamiento. La Organización de Estados Americanos (OEA) (2015) los comprende de la siguiente manera.

a) Activo Humano: habilidades, conocimiento y capacidades de trabajo.

b) Activo Social: vínculos y redes con individuos, instituciones o agrupaciones desde la confianza, reciprocidad e intercambio.

c) Activo Natural: riqueza natural, paisajística y bienes comunes de los territorios.

d) Activo Físico: bienes necesarios para el desarrollo económico, social y cultural.

e) Activo Financiero: ahorros, salarios, remesas o transferencia estatal.

2. Bienestar de las personas: la Fundación Superación de la Pobreza (2013) comprende el bienestar como mínimos satisfactores para mejorar la calidad de vida. Estos ámbitos son los explicados de la siguiente forma:

a) Educación: proceso de fortalecimiento de capacidades cognitivas, habilidades, valores, costumbres, físicas y emocionales. Es el fortalecimiento del capital humano y social.

Romero y Albornoz (2013), indican que la educación es base para enfrentar riesgos, sin embargo, actualmente se reduce a prácticas de comportamiento y sus establecimientos son usados como recurso físico en etapa de respuesta. En cambio, en la GRRD se concibe de la siguiente manera:

"El rol de la educación formal e informal es importante, debe contribuir a generar sensibilidad, debe anteceder la preocupación por acceder a información, conocer acciones necesarias e iniciar procesos de preparación; imprescindible para disminuir vulnerabilidad de la población local".

(Romero \& Albornoz, 2013, p. 522-523) 
b) Trabajo: acción creativa, preserva identidad, despliega solidaridad, utiliza experiencia organizativa y saber popular, satisface necesidades individuales y colectivas (Max-Neef, 1998).

c) Hábitat: asentamiento territorial, refugio, permite trasladarse, interactuar y conectarse.

d) Salud: estado de bienestar físico, psicológico y social que permite desarrollo de la vida.

e) Participación: inclusión en espacios sociales, en procesos significativos, siendo parte en decisiones que atañen directamente.

3. Autoprotección: se entendió en relación a la subsistencia y los medios de las personas y familias frente a riesgos. La DGPM-MEF (2007), indica tres factores necesarios a analizar:

a) Grado de Exposición: comprendido como riesgo latente. Es la proximidad a una amenaza. Los entornos riesgosos se ubican en contextos vulnerables, sus habitantes cuentan con disminuidos recursos y se considera como factor activador del desastre.

b) Fragilidad: particularidades del territorio relacionadas a tipo, cantidad y calidad de recursos, característica de activos que conforman los medios de vida de hogares y comunidades.

c) Resiliencia: capacidad de recuperación frente a desastres, expresado desde adaptación y absorción del impacto. En relación a lo anterior, Albornoz y Rodríguez (2013b) opinan que las organizaciones de Putre, son recursos sociales de acción colectiva y resiliencia comunitaria.

4. Protección Social: comprendida como un derecho. El Artículo No1 de la Constitución Política de la República de Chile (República de Chile, 1980) señala, "Es deber del Estado dar protección a la población y familia y salvaguardar el desarrollo del individuo y de la sociedad" (DS 100, 2005, art. 1). Asimismo, Mendizábal (2015) expresa que los beneficios estatales deben ser otorgados oportunamente, debiendo entregarse desde la etapa preventiva. Por lo demás, las instituciones chilenas han respondido al derecho constitucional, mediante el sistema nacional de emergencias y protección civil, destacando etapas del ciclo de la GRRD, que son: construcción, planificación, contingencia y emergencia.

La protección social ante emergencias conlleva un análisis situacional de comunidades, donde los servicios públicos, privados y sociedad civil conforman una red de colaboración. En el contexto de la región de Arica y Parinacota, la Fundación Superación de la Pobreza (2016) refiere que a través del Plan de Desarrollo de Zonas Extremas (PEDZE) ${ }^{2}$, se han construido diferentes estructuras mitigadoras de riesgo, como: conexión vial y sistema sanitario.

5. Gobernanza: apropiación comunitaria. El AVC lo define como un proceso de aplicación de soberanía popular. En la GRRD existen espacios de análisis de riesgos locales, instancia que contribuye a una adecuada protección social (FICR, 2006). Incluso la Organización de Naciones Unidas para la Reducción del Riesgo de Desastres (UNISDR) expuso como eje prioritario el fortalecimiento de la gobernanza civil. El Marco de Sendai (2015) faculta a los gobiernos locales para diseñar sistemas reguladores y financieros coordinados con la sociedad civil.

Albornoz y Rodríguez (2013a) señalan que las políticas chilenas en reducción del riesgo de desastres no cuentan con el énfasis social. Actualmente tienen una marcada lógica reactiva, impidiendo un trabajo colaborativo en etapas preventorias y mitigadoras. Los Planes de Protección Civil y emergencia comunal, tienen énfasis en la coordinación territorial.

a) Plan de protección civil: regula la estructura institucional de GRRD en diferentes niveles. Su objetivo es responder ante posibles emergencias o desastres. Por lo tanto, cuenta con instancias participativas de planificación. Por un lado, el Comité de Protección Civil, que coordina a públicos y privados, presidido por la autoridad local del ministerio del Interior y facultado para fijar normas relacionadas a GRRD. Por otro, el Comité de Operaciones de Emergencia (COE), constituido en etapa de emergencia, encargado de logística, conectividad, coordinación y comunicación (ONEMI, 2002).

\section{METODOLOGÍA}

Con el propósito de responder a la pregunta de investigación y a los objetivos instaurados, se trabajó mediante una investigación de indagación cualitativa (Taylor \& Bogdan, 1992). Lo que permitió estudiar un territorio y comprender las percepciones de los miembros del comité de protección civil
Plan de Desarrollo de Zonas extremas (PEDZE). Plan especial de intervención del GORE, reconoce la existencia de brechas que condicionan el desarrollo de los territorios, donde se necesitan políticas específicas de intervención (Gobierno Regional, 2017). 
y líderes vecinales de Putre. La pregunta se planteó mediante la construcción social del riesgo desde capacidades y vulnerabilidades que poseen las personas ante amenazas hidrometeorológicas, permitiendo indagar en experiencias pasadas, presentes y futuras en relación a percepción que aportan informantes en relación al riesgo.

A continuación, se presentan características del estudio, muestra, técnicas de producción y análisis de datos

Características del Estudio de Caso: la comuna de Putre, pertenece a Región de Arica y Parinacota, tiene una superficie de $5.902,5 \mathrm{~km}^{2}$. Además se encuentra entre dos pisos ecológicos de precordillera (2500 a $4000 \mathrm{msnm}$ ) y altiplano (sobre $4000 \mathrm{msnm}$ ). También, un $70 \%$ de la comuna corresponde a áreas protegidas (Ilustre Municipalidad de Putre, 2014).

La población comunal, según CENSO 2017, es de 2.765 habitantes (INE, 2017). En medición sobre pobreza, tiene un $22,5 \%$ de población bajo la línea de pobreza por ingreso y un $58 \%$ en pobreza multidimensional (MDS, 2013). Putre, se encuentra expuesta a amenazas de precipitaciones, con riesgos de inundación, socavones, corte viales y comunicación.

Selección de Participantes: uso de muestra intencionada (Flick, 2007). Debido a la pregunta de investigación se delimitó a personas del comité de protección civil y líderes vecinales. Su selección se debió al conocimiento territorial y relaciones. Se utilizaron dos escalas de estudio. La unidad de observación, conformada por dirigentes y representantes institucionales. Además, la unidad espacial de análisis, referida a la comuna de Putre y localidades de Tignamar y Belén, territorios expuestos a precipitaciones estivales.

Caracterización Muestral: separada en dirigentes sociales de localidades rurales y urbanas. Compuesta de 2 representantes municipales, 2 personas de la sociedad civil organizada como el Comité de agua potable rural (APR) y 6 dirigentes pertenecientes a Junta de Vecinos (JJVV) Comunidad Indígena y Club de Adulto Mayor.

Técnicas de Producción de Datos: Durante Agosto de 2016, se planteó una estrategia en relación a tipos de participantes (Flick, 2007), creando instrumentos que respondieron al tipo de entrevistados. A continuación, se describe la entrevista Semiestructurada, como una técnica que permite comprender las percepciones, describiendo oralmente su sentir y conocimiento del fenómeno a investigar (Mayan, 2001). En el instrumento se utilizaron preguntas abiertas, considerando la información que se requería. Estos fueron grabados, transcritos y analizados. Igualmente, se arma una matriz de Análisis de Datos, sobre dimensiones de vulnerabilidad y capacidades, donde los datos son categorizados. Los componentes utilizados son de Federación Internacional de Sociedades de la Cruz y Media Luna Roja (FICR). Estos son: a) Medios de subsistencia, b) Bienestar de las personas, c) Autoprotección, d) Protección Social y c) Gobernanza. Para su análisis categorial se confeccionó una matriz de categorías y subcategorías, permitiendo ordenar y encontrar hallazgos en el estudio.

\section{RESULTADOS}

El estudio se realizó por medio de 'Observación de Conceptos' del método AVC, durante el año 2016. La pregunta se planteó en base a que los riesgos son una construcción social, instaurada desde capacidades y contextos de vulnerabilidad. La construcción social permitió resaltar subjetividades, indagando en experiencias pasadas, presentes y futuras en relación al ciclo del riesgo. Las percepciones comunitarias fueron organizadas en capacidades y vulnerabilidades. Además, fueron analizadas en cinco categorías descritas en la metodología AVC: 1.Medios de subsistencia, 2.Bienestar de las personas, 3.Autoprotección, 4.Protección social y 5.Gobernanza; destacando conceptos emergentes desde el discurso de los entrevistados.

\section{Categoría Medios de Subsistencia y Capacidad de Resistencia, capacidades y vulnerabilidades}

Se entiende en relación a cinco activos necesarios para el desarrollo comunitario. Kaztman y Filgueira (1999) indican que los recursos deben conectarse a cierta estructura para que ser activados y sostenibles comunitariamente, satisfaciendo necesidades.

1. Subcategoría Activo Humano: se observaron diferencias entre lo rural y urbano. En lo rural, lo relacionaron a personas que habitan los territorios, que son participativos en etapa de emergencia, destacando a funcionarios públicos como parte de la comunidad activa y preparada (paramédicos 
y profesores). En contradicción, lo urbano que observan a instituciones como principal recurso, debido a su cercanía. Además, percibieron vulnerabilidades en el ciclo de GRRD, entre estas, falta de preparación, capacitación y ayuda material (Post emergencia). Conjuntamente, la institucionalidad local cuenta con una disminuida dotación profesional, donde surge duplicidad de tareas y solo respuesta a emergencias. Cabe señalar que no han desarrollado GRRD a nivel local. A continuación conceptos saturados.

\begin{tabular}{|c|c|c|}
\hline Subcategoría & Vulnerabilidades (-) & Capacidades (+) \\
\hline \multirow{4}{*}{ 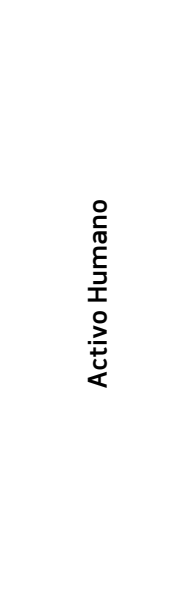 } & Área Rural & \\
\hline & $\begin{array}{l}\text { - Falta de diversidad etaria } \\
\text { - No cuentan con conocimiento para ha- } \\
\text { cer frente a emergencia (Ej:. } 1^{0} \text { Auxilios) } \\
\text { - Reactividad institucional }\end{array}$ & $\begin{array}{l}\text { - Existencia de jóvenes que apoyan a otros } \\
\text { - Dirigentes comunicados con instituciones } \\
\text { en etapa post } \\
\text { - Catastro de comunidad a autoridades } \\
\text { - Dirigentes conocen a encargado de } \\
\text { emergencias }\end{array}$ \\
\hline & Área Urbana & \\
\hline & $\begin{array}{l}\text { - Falta diversidad etaria } \\
\text { - Desconocen a encargado de } \\
\text { emergencia } \\
\text { - No existen roles y preparación en GRRD } \\
\text { - Instituciones reactivas }\end{array}$ & $\begin{array}{l}\text { - Visualizan a instituciones activas } \\
\text { (Militares y carabineros) en etapa post } \\
\text { - Participan en instancias intersectoriales } \\
\text { post emergencias (COE) }\end{array}$ \\
\hline
\end{tabular}

Tabla 1. Percepción del Activo Humano, Capacidades y Vulnerabilidades por sector rural y urbano Fuente: Autora, 2018

2. Subcategoría Activo Social: en área rural y urbana es percibido como organización, con características de reciprocidad, que irrumpen en instancias que afectan a la comunidad. Desde lo rural expresaron que en post emergencia, se unen y olvidan rencillas. Asimismo, la institución municipal reconoció a la comunidad rural, debido a su organización. De esta manera, el dirigente cumplió un rol importante en levantar el catastro preliminar y en comunicar al COE, con el fin de conseguir ayuda. Del mismo modo, la comunidad realiza limpieza preventiva de canales en época de precipitaciones.

Por consiguiente, se enseña aquellas vulnerabilidades y capacidades, que emergen del discurso de las personas entrevistadas.

\begin{tabular}{|c|c|c|}
\hline Subcategoría & Vulnerabilidades (-) & Capacidades (+) \\
\hline \multirow{4}{*}{ 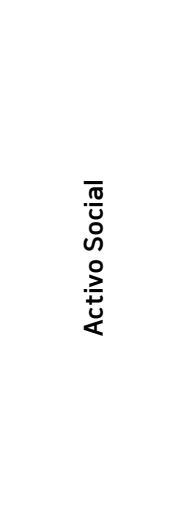 } & Área Rural & \\
\hline & $\begin{array}{l}\text { - Falta diversidad etaria } \\
\text { - Existe desconfianza entre dirigentes } \\
\text { - Alta población de adultos mayores. }\end{array}$ & $\begin{array}{l}\text { - Conocimiento de organizaciones locales } \\
\text { - Organización y colaboración entre vecinos } \\
\text { - Reactivación en caso de emergencias y } \\
\text { protección de bienes comunes } \\
\text { - Predominan acciones ancestrales de } \\
\text { reciprocidad }\end{array}$ \\
\hline & Área Urbana & \\
\hline & $\begin{array}{l}\text { - Desconocimiento en GRRD } \\
\text { - Descoordinación en etapas de GRRD } \\
\text { - Falta diversidad etaria en socios }\end{array}$ & $\begin{array}{l}\text { - Dirigentes mantienen alerta y colaboran } \\
\text { con demás vecinos } \\
\text { - Acciones comunitarias para proteger } \\
\text { bienes comunes (cementerio y canales) }\end{array}$ \\
\hline
\end{tabular}

Tabla 2. Percepción del Activo Social, Capacidades y Vulnerabilidades por sector

Fuente: Autora, 2018

3. Subcategoría Activo Natural: personas desde lo rural y urbano percibieron bienes comunes como principal activo, por ejemplo flora, fauna y paisaje. Al mismo tiempo, expresaron que las lluvias estivales se convierten en problema, cuando remueven lodo desde quebradas, arrasan conectividad vial, dejan de nutrir bofedales y afectan canales de regadío. Sin embargo, las personas observaron que las precipitaciones cada año fluctúan, pudiendo provocar inundaciones o sequías, 
interfiriendo en el ciclo productivo ganadero y agrícola.

De igual manera, en el territorio existieron relatos históricos sobre ríos caudalosos, que había provocado aislamiento y arrasado con poblados completos. En la posterior tabla, se visualiza capacidades y vulnerabilidades.

\begin{tabular}{|c|c|c|}
\hline Subcategoría & Vulnerabilidades (-) & Capacidades (+) \\
\hline \multirow{4}{*}{ 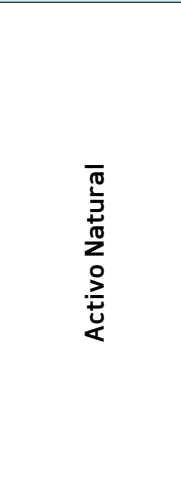 } & Área Rural & \\
\hline & $\begin{array}{l}\text { - Aumento de caudal } \\
\text { - Aludes y socavones } \\
\text { - Inundaciones y sequía }\end{array}$ & $\begin{array}{l}\text { - Lluvia nutre suelo agrícola } \\
\text { - Aumento reserva nival en montañas } \\
\text { - Presencia de flora y fauna nativa } \\
\text { - Agricultura de subsistencia }\end{array}$ \\
\hline & Área Urbana & \\
\hline & $\begin{array}{l}\text { - Cercanía de complejo volcánico } \\
\text { - Aumento de caudal } \\
\text { - Aludes y socavones } \\
\text { - Inundaciones y Sequía }\end{array}$ & $\begin{array}{l}\text { - Aumento reserva nival en montañas } \\
\text { - Presencia de Flora y Fauna nativa } \\
\text { - Agricultura de subsistencia }\end{array}$ \\
\hline
\end{tabular}

Tabla 3. Percepción del Activo Natural, Capacidades y Vulnerabilidades por sector Fuente: Autora, 2018

4. Subcategoría Activo Físico: desde lo rural y urbano se entendió como infraestructura comunitaria para protección. Conjuntamente, han sido mejoradas y construidas con fondos estatales PEDZE.

Asimismo, la comunidad diferenció dos tipos de activos. Por un lado, el comunitario que ha sido mejorado y construído con fondos estatales. Por el otro, el familiar donde el hogar accede a fondos propios y comerciales para mejorar viviendas, previo a temporada de lluvias. En el próximo apartado, una tabla con vulnerabilidad y capacidad en obras físicas.

\begin{tabular}{|c|c|c|}
\hline Subcategoría & Vulnerabilidades (-) & Capacidades (+) \\
\hline \multirow{4}{*}{ 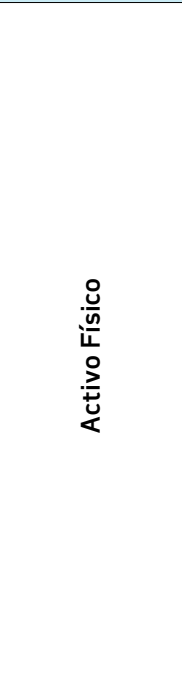 } & Área Rural & \\
\hline & $\begin{array}{l}\text { - Presencia de obras peatonales } \\
\text { - No existe mejoramiento de Badenes } \\
\text { - No existe respeto en técnicas ancestra- } \\
\text { les de mejoramiento vial }\end{array}$ & $\begin{array}{l}\text { - Conocimiento en infraestructura } \\
\text { comunitaria } \\
\text { - Construcción y mejoramiento con fondos } \\
\text { PEDZE } \\
\text { - Conexión vial } \\
\text { - Mejoramiento infraestructura familiar } \\
\text { por fundaciones } \\
\text { - Mejoramiento de abastecimiento de APR. }\end{array}$ \\
\hline & Área Urbana & \\
\hline & $\begin{array}{l}\text { - Sin defensa fluvial } \\
\text { - Remoción de suelo en cementerio } \\
\text { - Sin tecnologías como radios o } \\
\text { megáfono } \\
\text { - Sin señalética (área de seguridad y } \\
\text { albergue) }\end{array}$ & $\begin{array}{l}\text { - Arreglos y preparación de infraestructura } \\
\text { familiar } \\
\text { - Cuentan con maquinarias municipales y } \\
\text { de FF.AA }\end{array}$ \\
\hline
\end{tabular}

Tabla 4. Percepción Activo Físico, Capacidades y Vulnerabilidades por sector Fuente: Autora, 2018

5. Subcategoría Activo Financiero: las áreas rurales y urbanas, lo entienden como ahorro, préstamo o subsidio. Con la finalidad de mejorar las condiciones de habitabilidad y producción. Generalmente de origen estatal, siendo obtenidas mediante postulación a proyectos o desde el pertenecer a un tramo del $\mathrm{RSH}^{3}$. Por consiguiente, en la etapa post en GRRD, las personas pueden acceder a estos beneficios. En etapa preventiva deben recurrir a ahorros o préstamos comerciales. A continuación, presentamos capacidades y vulnerabilidad.

3. El Registro Social de Hogares (RSH) Es una base de datos que contiene información de personas y hogares. Sirv para apoyar la postulación y selección de las personas a diversos beneficios sociales estatales, acceso a subsidios de reconstrucción y mejoramiento (Ministerio de Desarrollo Social, 2017) 


\begin{tabular}{|c|c|c|}
\hline Subcategoría & Vulnerabilidades (-) & Capacidades (+) \\
\hline \multirow{4}{*}{ 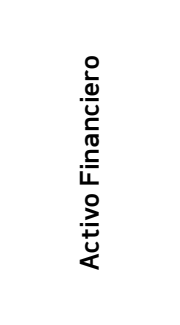 } & Área Rural & \\
\hline & - No visualiza acceso a recursos propios & - Financiamiento de instituciones estatales \\
\hline & Área Urbana & \\
\hline & $\begin{array}{l}\text { - No pueden acceder a fondos estatales } \\
\text { para mejorar bienes comunes } \\
\text { - No pueden acceder a fondos en etapas } \\
\text { preventivas }\end{array}$ & $\begin{array}{l}\text { - Subsidio mejoramiento techumbre y } \\
\text { fachada } \\
\text { - Uso de recursos propios para mejorar } \\
\text { vivienda en etapa pre y post emergencia }\end{array}$ \\
\hline
\end{tabular}

Tabla 5. Percepción del Activo Financiero, Capacidades y Vulnerabilidades por sector Fuente: Autora, 2018

\section{Categoría Bienestar de las personas}

Las personas mejoran su calidad de vida, si cuentan con mínimos garantizados en su bienestar. Esta categoría, buscó profundizar las percepciones sobre los cinco ámbitos de bienestar y los satisfactores utilizados desde el punto de vista sobre GRRD.

1. Subcategoría ámbito Educación: desde el área rural y urbana, fue entendida como conocimiento en GRRD y formación comunitaria. Es un proceso de creación de capital social y humano, para enfrentar riesgos. En ambas espacios, las personas destacan vulnerabilidades, como desconocimiento en GRRD. Opinaron, no vincularse en etapas previas del ciclo del riesgo con instituciones. Los representantes institucionales, indican que los establecimientos educativos son utilizados como albergue, revelan una inexistencia de planes de emergencia escolares. De esta forma, los entrevistados no observan capacidades. Desde las vulnerabilidades se piensan posibles talleres de sensibilización.

\begin{tabular}{|c|c|c|}
\hline Subcategoría & Vulnerabilidades (-) & Capacidades (+) \\
\hline \multirow{4}{*}{ 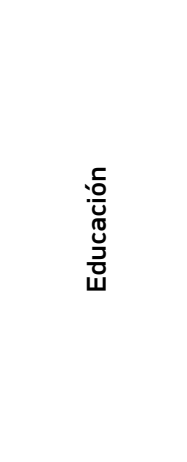 } & Área Rural & \\
\hline & $\begin{array}{l}\text { - Falta de capacitaciones en GRRD } \\
\text { - Descontextualización de capacitaciones }\end{array}$ & \\
\hline & Área Urbana & \\
\hline & $\begin{array}{l}\text { - Desconocimiento en planes de emergencia } \\
\text { municipal } \\
\text { - Falta de capacitaciones en GRRD } \\
\text { - Falta de socialización de acciones institucionales } \\
\text { - Inactividad de instituciones en etapa preventiva } \\
\text { del ciclo de GRRD }\end{array}$ & \\
\hline
\end{tabular}

Tabla 6. Percepción ámbito de Bienestar Educación, Capacidades y Vulnerabilidades por sector Fuente: Autora, 2018

2. Subcategoría ámbito Trabajo: desde el área rural y urbana reconocieron las implicancias de riesgos en actividades económicas. Las personas visualizaron tres ejes producticos, entre ellos turismo, ganadería y agricultura. En consecuencia, una de las más afectadas es la agropecuaria, revelando tecnologías inapropiadas para el territorio. De este modo, las precipitaciones aquejan producciones de alfalfa, situación que afecta la ganadería. Por otra parte, aquellos productores que alcanzan a cosechar, no podían vender sus productos, debido a cortes viales e inaccesibilidad de puntos de venta en la comuna de Arica.

\begin{tabular}{|c|c|c|}
\hline Subcategoría & Vulnerabilidades (-) & Capacidades (+) \\
\hline \multirow[b]{2}{*}{$\begin{array}{l}\stackrel{ }{\pi} \\
\frac{0}{0} \\
\text { 은 }\end{array}$} & Área Rural y Urbana & \\
\hline & $\begin{array}{l}\text { - Falta de comercialización } \\
\text { - Perdida en el ganado } \\
\text { - Disminuye la producción } \\
\text { - Afectación en canalización (bocatomas) } \\
\text { - Perdida de suelo y humedales } \\
\text { - Efectos en producción forrajera } \\
\text { - Tecnologías inapropiadas } \\
\text { - Inequidad en acceso financiero }\end{array}$ & $\begin{array}{l}\text { - Acceso estatal para mejorar producción } \\
\text { - Tecnologías para producir en precordillera } \\
\text { - Desarrollo y trabajo conjunto de áreas } \\
\text { económicas }\end{array}$ \\
\hline
\end{tabular}

4. Se entenderá al mediero como figur legal, según un contrato de arriendo, donde una parte se obliga a aportar el uso de una superficie de terreno y la otra el trabajo para realizar cultivos determinados, con el objeto de repartirse la producción resultante (Ley 993 , 1975, art 12). 
Conjuntamente, las personas acceden a apoyo estatal en estas circunstancias, oportunidades usadas en rescate productivo. Sin embargo, los medieros ${ }^{4}$ de suelos agrícolas, no pueden acceder a estos subsidios, provocando una pérdida total o considerable de su cosecha. La Tabla 7 (arriba) resume las capacidades y vulnerabilidades en ámbito trabajo.

3. Subcategoría ámbito Hábitat: las áreas rural y urbana, lo entendieron desde entorno habitable y riesgo hidrometeorológico. Asimismo, se observó dos entornos afectados. El natural, donde existe afectación del suelo y aumento del caudal y hábitat construido, con efectos viales, destrucción de canales y bocatomas. Las precipitaciones estivales, son visualizadas como recurso importante. Las personas, reconocen que afecta el forraje, pero es beneficiosa en el desarrollo de actividad agrícola y ganadera. Sin embargo, si son abundantes e intensas (frecuencia y duración) pueden convertirse en amenaza para las comunidades. A continuación el subcriterio evaluado.

\begin{tabular}{|c|c|c|}
\hline Subcategoría & Vulnerabilidades (-) & Capacidades (+) \\
\hline \multirow{4}{*}{$\begin{array}{l}\stackrel{+}{0} \\
\stackrel{ \pm}{0} \\
\frac{0}{ \pm}\end{array}$} & Área Rural & \\
\hline & $\begin{array}{l}\text { - Presencia de plagas } \\
\text { - Inundaciones de suelos } \\
\text { - Corte vial y canalización } \\
\text { - Calidad de agua } \\
\text { - Aislamiento }\end{array}$ & $\begin{array}{l}\text { - Conocimiento de amenazas } \\
\text { - Socializan ante instituciones } \\
\text { - Precipitaciones como un recurso agrícola } \\
\text { y ganadero }\end{array}$ \\
\hline & Área Urbana & \\
\hline & $\begin{array}{l}\text { - Cercanía a complejo vulcanológico } \\
\text { - Inundaciones } \\
\text { - Territorio inseguro } \\
\text { - Corte vial y canalización } \\
\text { - Calidad de agua } \\
\text { - Acceso } \\
\text { - Aislamiento } \\
\text { - Desconexión con Arica }\end{array}$ & $\begin{array}{l}\text { - Conocimiento de amenazas } \\
\text { - Socializan ante instituciones } \\
\text { - Precipitaciones son un recurso agrícola y } \\
\text { ganadero }\end{array}$ \\
\hline
\end{tabular}

Tabla 8. Percepción del ámbito de Bienestar Hábitat, Capacidades y Vulnerabilidades por sector Fuente: Autora, 2018

4. Subcategoría ámbito Salud: desde lo rural y urbano, es analizado desde la institucionalidad y su relación comunitaria. Por consiguiente, el área rural describió una relación con encargados de la posta. Indicando ser activos en emergencia, colaborando en $1^{\circ}$ auxilios y comunicación. Igualmente, expresaron el desarrollo de capacitaciones, que no fueron de interés comunitario, por poco prácticas. En cambio, en lo urbano no cuentan con vínculo en GRRD, opinando que el acercamiento es en etapa de emergencia. Las instituciones, reconocieron inexistencia de planes orientadores conjuntos. A continuación la información proporcionada.

\begin{tabular}{|c|c|c|}
\hline Subcategoría & Vulnerabilidades (-) & Capacidades (+) \\
\hline \multirow{4}{*}{$\frac{\bar{\partial}}{\frac{\partial}{\pi}}$} & Área Rural & \\
\hline & $\begin{array}{l}\text { - Traslado del paciente } \\
\text { - Problemas de salud en personas de } \\
3^{\circ} \text { edad. } \\
\text { - Falta de talleres prácticos en } 1^{\circ} \text { auxilios } \\
\text { - Problemas en rondas médicas }\end{array}$ & $\begin{array}{l}\text { - Vínculo con paramédicos } \\
\text { - Forman parte del COE } \\
\text { - Comunican estado comunitario } \\
\text { - Charlas comunitarias } \\
\text { - Interés en capacitaciones en } 1^{\circ} \text { auxilios }\end{array}$ \\
\hline & Área Urbana & \\
\hline & $\begin{array}{l}\text { - Sin vínculo en GRRD } \\
\text { - No imparten talleres prácticos }\end{array}$ & - Pertenecer al comité consultivo comunal \\
\hline
\end{tabular}

Tabla 9: Percepción del ámbito de Bienestar Salud, Capacidades y Vulnerabilidades por sector Fuente: Autora, 2018

5. Subcategoría ámbito Participación: en área rural y urbana es comprendido desde la inclusión en toma de decisiones. De esta manera, destaca un arraigo en la cultura aymara, que ancestralmente participativa en acciones comunitarias, destacándose reciprocidad. Asimismo, opinan que 
han sido afectados por las políticas, debido a su fortalecimiento individual. Por lo consiguiente, desde lo urbano existe una mayor aproximación en estructura de oportunidades (EEOO) ${ }^{5}$, recibiendo colaboración. En cambio, en lo rural han tenido que hacer frente a los riesgos, mediante trabajo comunitario en prevención y reconstrucción, por ejemplo, mejorando viviendas, limpiando canales y bocatomas. A continuación tabla con la diferenciación entre rural y urbano.

\begin{tabular}{|c|c|c|}
\hline Subcategoría & Vulnerabilidades (-) & Capacidades (+) \\
\hline \multirow{4}{*}{ 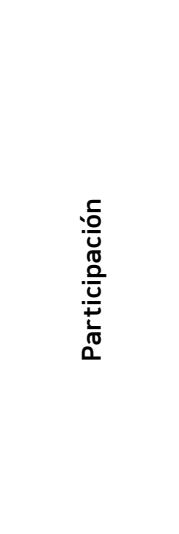 } & Área Rural & \\
\hline & $\begin{array}{l}\text { - Poca diversidad etaria } \\
\text { - Conflictos comunitarios }\end{array}$ & $\begin{array}{l}\text { - Colaboración entre vecinos } \\
\text { - Prácticas ancestrales } \\
\text { - Organizaciones ante emergencia } \\
\text { - Gestión de soluciones } \\
\text { - Catastro ante instituciones } \\
\text { - Vínculo y presencia de redes sociales }\end{array}$ \\
\hline & Área Urbana & \\
\hline & $\begin{array}{l}\text { - Disminuye trabajo comunitario } \\
\text { - Hermetismo institucional } \\
\text { - Sin espacios de vinculación } \\
\text { - Conflictos comunitarios }\end{array}$ & $\begin{array}{l}\text { - Gestión comunitaria, denominada } \\
\text { Delegación de aguas } \\
\text { - Prácticas en mejoramiento de caminos } \\
\text { y canales }\end{array}$ \\
\hline
\end{tabular}

Tabla 10. Percepción del ámbito Participación, Capacidades y Vulnerabilidades por sector Fuente: Autora, 2018

\section{Categoría de Autoprotección}

Entendida como cualidades para hacer frente a riesgos. Asimismo, hace referencia a recursos comunitarios y familiares. De este modo, fortalecer las características de adaptación, es una forma de hacer frente a los desastres.

Putre es afectada anualmente por precipitaciones, acentuando el uso de prácticas ancestrales en las áreas de menor influencia de la EEOO. A continuación, ahondaremos en los tres conceptos que explican la categoría.

1. Subcategoría Grado de Exposición: desde el área rural y urbana, se comprende como el activador del desastre, intensificado en contextos vulnerables. De esta manera, en ambas áreas las personas tienden a buscar explicaciones teológicas ante riesgos. No vinculan el riesgo, con habitar entornos vulnerables. Asimismo, conocen su territorio en relación a sus experiencias, destacando relatos sobre inundaciones, socavones y deslizamiento en quebradas. Por tanto, admiten que Putre se ubica en presencia de amenazas que los pueden afectar. A continuación, principales capacidades y vulnerabilidades.

\begin{tabular}{|c|c|c|}
\hline Subcategoría & Vulnerabilidades (-) & Capacidades (+) \\
\hline \multirow{4}{*}{$\begin{array}{l}: 0 \\
: \frac{0}{U} \\
\frac{0}{0} \\
\frac{0}{x} \\
x \\
\frac{0}{0} \\
\frac{0}{0} \\
\frac{\pi}{0}\end{array}$} & Área Rural & \\
\hline & $\begin{array}{l}\text { - Caudales esporádicos } \\
\text { - Desplazamiento de tierra } \\
\text { - Cortes viales } \\
\text { - Adobe de viviendas } \\
\text { - Mayor afectación de adultos mayores } \\
\text { - Impacto en productividad } \\
\text { - Explicación teológica }\end{array}$ & $\begin{array}{l}\text { - Viviendas en áreas seguras } \\
\text { - Acceso a obras de resguardo }\end{array}$ \\
\hline & Área Urbana & \\
\hline & $\begin{array}{l}\text { - Territorio inundable } \\
\text { - Amenazas naturales. (volcán, lluvias y temblores) } \\
\text { - Aislamiento } \\
\text { - Afectación de bien común } \\
\text { - Construcción en pendiente }\end{array}$ & \\
\hline
\end{tabular}

5. Estructura de Oportunidades, desde ahora abreviado como EE.OO. Definida como probabilidades de acceso a bienes, a servicios o al desempeño de actividades. Inciden en el bienestar de los hogares, ya que facilita el uso d recursos (Kaztman \& Filgueira, 1999). 
2. Subcategoría Fragilidad: en el área rural y urbana se comprendió desde los medios de vida, es una condición de inestabilidad de recursos que cuentan las personas en su entorno. De esta manera, se centra sobre el territorio, donde una conexión a una EEOO pertinente, disminuye la variable. Igualmente, las personas indican diferentes causas de fragilidad, una de estas es el cambio climático. Fenómeno, debido a la concentración del $\mathrm{CO}_{2}$, que produce aumento de temperatura en la superficie terrestre, provocando impactos en el clima (Convención Marco de las Naciones Unidas sobre el Cambio Climático (2016).

Por tanto, las personas expresan dificultad de hacer frente a estos cambios, por estar fuera de su alcance. Estos describen positivamente prácticas comunitarias preventivas. La subcategoría es comprendida en relación a los siguientes conceptos, permitiendo observarla un contexto geográfico.

\begin{tabular}{|c|c|c|}
\hline Subcategoría & Vulnerabilidades (-) & Capacidades (+) \\
\hline \multirow{4}{*}{ 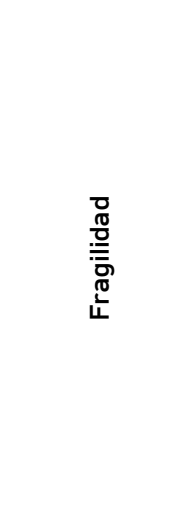 } & Área Rural & \\
\hline & $\begin{array}{l}\text { - Fragilidad Ambiental } \\
\text { - Sistema de agua potable } \\
\text { - Aumento de caudal } \\
\text { - Viviendas ribereñas } \\
\text { - Presencia de Plagas } \\
\text { - Desvinculación con instituciones }\end{array}$ & $\begin{array}{l}\text { - Manejo sobre cambio climático y sus } \\
\text { consecuencias } \\
\text { - Responsabilidad con el entorno }\end{array}$ \\
\hline & Área Urbana & \\
\hline & $\begin{array}{l}\text { - Geografía en forma de hendidura. } \\
\text { - Desvinculación entre vecinos e } \\
\text { instituciones } \\
\text { - Diversidad de amenazas }\end{array}$ & $\begin{array}{l}\text { - Manejo sobre cambio climático y sus } \\
\text { consecuencias }\end{array}$ \\
\hline
\end{tabular}

Tabla 12. Percepción de Fragilidad, Capacidades y Vulnerabilidades, por sector rural y urbano Fuente: Autora, 2018

3. Subcategoría Resiliencia: desde el área rural y urbano es visto como la capacidad de recuperación de personas y comunidades. De esta forma, se analiza desde la preparación comunitaria ante posibles riesgos, revalorizando prácticas desarrolladas.

Asimismo, en lo rural se aprecian prácticas relacionadas a la cultura aymara, como la reciprocidad o ayni. Desde lo urbano, indican vincularse con los vecinos, ante la emergencia, que es un precursor de acciones en conjunto. Se entiende, que las prácticas ancestrales tienen una fuerte relación con sus sistemas productivos, por ejemplo: la limpieza de canales, busca solucionar la repartición de agua.

Además, las instituciones destacan la existencia de organizaciones, donde el Presidente de la JJ.VV da el catastro preliminar, teniendo un alto conocimiento de afectaciones, posibilitando la colaboración. A continuación, prácticas resilientes comunitarias.

\begin{tabular}{|c|c|c|}
\hline Subcategoría & Vulnerabilidades (-) & Capacidades (+) \\
\hline \multirow{4}{*}{ 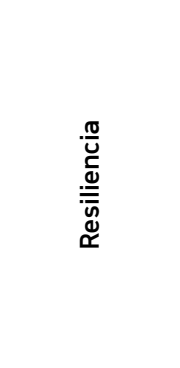 } & Área Rural & \\
\hline & $\begin{array}{l}\text { - Disminuida población } \\
\text { - Falta diversidad etaria }\end{array}$ & $\begin{array}{l}\text { - Prácticas ancestrales } \\
\text { - Trabajos comunitarios colaborativos }\end{array}$ \\
\hline & Área Urbana & \\
\hline & $\begin{array}{l}\text { - Falta diversidad etaria } \\
\text { - Asistencia del gobierno } \\
\text { - Delegación de acciones comunitarias } \\
\text { - Pérdida de memoria histórica }\end{array}$ & $\begin{array}{l}\text { - Trabajos comunitarios colaborativos, } \\
\text { desde delegación de aguas }\end{array}$ \\
\hline
\end{tabular}

Tabla 13. Percepción de Resiliencia, Capacidades y Vulnerabilidades, área rural y urbano Fuente: Autora, 2018

\section{Categoría Protección Social}

Mínimos de bienestar para enfrentar y reconstruir medios de vida, después de un desastre. Son tres derechos que debe proporcionar el Estado, para que familias satisfagan sus necesidades como: vivienda, salud y seguridad de medios de vida en GRRD. De la misma forma, Kaztman y 
Filgueira (1999) indican que las familias y comunidades son las que proporcionan un núcleo básico de protección y seguridad ante riesgos. Para su análisis se revisaron tres componentes de protección Social ante riesgos de desastre.

1. Subcategoría Vivienda: desde lo urbano y rural, es entendida como protección para las personas. En ambos, los individuos hacen arreglos con recursos propios, siendo una estrategia para prevenir y mitigar impactos por las lluvias. Aun así, en el estudio se observan diferencias entre área rural y urbana.

Desde lo rural, el Estado mediante el trabajo de fundaciones ha mantenido casas patrimoniales, siendo una oportunidad para mejorar viviendas. Sin embargo, emergieron relatos sobre entrega de material inadecuado a las necesidades, pudiendo ser causa de problemas estructurales a largo plazo. Expresan que la asistencia municipal es baja, pero que algunos materiales son vistos como ayuda hacia las personas, que no pueden acceder a recursos financieros privados. Desde Putre urbano, indican contar con subsidio de reconstrucción, permitiendo mejorar su habitabilidad. En el siguiente cuadro se observa capacidades y vulnerabilidades.

\begin{tabular}{|c|c|c|}
\hline Subcategoría & Vulnerabilidades (-) & Capacidades (+) \\
\hline \multirow{4}{*}{$\underset{\substack{0 \\
\frac{1}{0}}}{\frac{\pi}{2}}$} & Área Rural & \\
\hline & $\begin{array}{l}\text { - Casas antiguas con problemas } \\
\text { constructivos } \\
\text { - Adsorción de humedad desde cimientos }\end{array}$ & $\begin{array}{l}\text { - Entrega de materiales estatales } \\
\text { - Técnicas constructivas ancestrales, mejo } \\
\text { ramiento propio } \\
\text { - Mejoramiento de fachadas por parte de } \\
\text { ONG }\end{array}$ \\
\hline & Área Urbana & \\
\hline & $\begin{array}{l}\text { - Entrega viviendas de emergencias que } \\
\text { pasan a ser definitivas }\end{array}$ & - Acceso a subsidios estatales \\
\hline
\end{tabular}

Tabla 14. Percepción sobre la Vivienda, Capacidades y Vulnerabilidades por sector Fuente: Autora, 2018

2. Subcategoría Salud: entendido como estado de bienestar del individuo y comunidad, permite un desarrollo de vida adecuado de acuerdo a las etapas del ciclo vital. Asimismo, en área rural y urbana, el grupo más afectado por diferentes enfermedades son los adultos mayores. Estos son tratados en rondas médicas y en postas, instancias valoradas comunitariamente. La tabla adjunta enseña conceptos emergentes del subcriterio salud.

\begin{tabular}{|c|c|c|}
\hline Subcategoría & Vulnerabilidades (-) & Capacidades (+) \\
\hline \multirow{4}{*}{$\frac{\bar{\partial}}{\sqrt{n}}$} & Área Rural & \\
\hline & $\begin{array}{l}\text { - Traslado paciente en caso de accidente } \\
\text { - Problemas de salud de las personas de } \\
3^{\circ} \text { edad } \\
\text { - Problemas en rondas por cortes de } \\
\text { camino }\end{array}$ & $\begin{array}{l}\text { - Vínculo con paramédicos } \\
\text { - Charlas básicas hacia la comunidad } \\
\text { - Interés en capacitaciones en salud }\end{array}$ \\
\hline & Área Urbana & \\
\hline & $\begin{array}{l}\text { - Falta de vínculo en emergencia y riesgo } \\
\text { - No imparten talleres prácticos } \\
\text { comunitarios }\end{array}$ & - Comité comunal \\
\hline
\end{tabular}

Tabla 15. Percepción sobre Salud, Capacidades y Vulnerabilidades por sector Fuente: Autora, 2018

3. Subcategoría Seguridad de los Medios de Vida: recursos de personas y familias para hacer frente a los desastres. Desde la protección se necesita una serie de regulaciones del espacio, como planes de ordenamiento territorial, estos se deben socializar para prevenir y dar respuesta a situaciones desastrosas. En esta subcategoría emergen diferencias como el desconocimiento de la construcción de planes de emergencia entre sociedad civil (desde lo rural y urbano) e instituciones. Ambas áreas rural y urbana, indican no conocer ningún tipo de planificación, debido a que no son partícipes en las etapas de construcción, percibiéndolas como necesarias en la regulación del hábitat para enfrentar amenazas. Del mismo modo, se aprecia la inexistencia de la prevención de riesgos en los planes municipales. A continuación, una tabla que ordena conceptos emergentes sobre capacidades y vulnerabilidades. 


\begin{tabular}{|c|c|c|c|}
\hline \multicolumn{2}{|c|}{ Subcategoría } & Vulnerabilidades (-) & Capacidades (+) \\
\hline \multirow{4}{*}{ 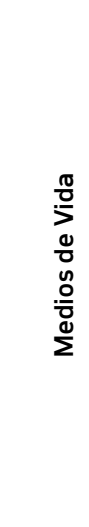 } & \multirow{4}{*}{ 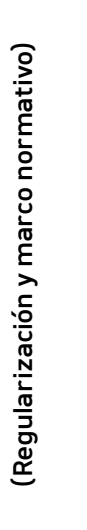 } & Área Rural & \\
\hline & & $\begin{array}{l}\text { - Falta de socialización de normativas } \\
\text { - Planes se hacen sólo en área urbana } \\
\text { de Putre } \\
\text { - Inexistencia de fiscalización }\end{array}$ & $\begin{array}{l}\text { - Disponibilidad de participación en regula- } \\
\text { rización del entorno }\end{array}$ \\
\hline & & Área Urbana & \\
\hline & & $\begin{array}{l}\text { - Desconocimiento de planes regulatorios } \\
\text { del entorno o en GRRD } \\
\text { - Falta socializar el marco normativo } \\
\text { - Desconocimiento de roles en } \\
\text { emergencia }\end{array}$ & $\begin{array}{l}\text { - Disponibilidad de participación en regula- } \\
\text { rización del entorno }\end{array}$ \\
\hline
\end{tabular}

Tabla 16. Percepción sobre Seguridad de los medios de vida, Capacidades y Vulnerabilidades Fuente: Autora, 2018

\section{Categoría Gobernanza.}

Entendido como apropiación comunitaria en GRRD. Es la vinculación de la ciudadanía en las diferentes escalas (local, regional y nacional). En relación a una catástrofe, la experiencia comunitaria es fundamental para comprender contextos habitados, propiciando protección social adecuada.

1. Subcategoría Organizaciones de la Sociedad Civil ante Gestión del Riesgo: en lo urbano indican falta de preparación, para hacer frente a desastres. De este modo, expresan no estar organizados en etapas preventivas del ciclo de GRRD, dando sólo respuesta ante emergencia. Expresan que la GRRD debe ser construida con la comunidad, proponiendo información pertinente al contexto. Desde lo rural, muestran una participación activa en la etapa post emergencia, organizándose vecinalmente, realizan catastros, tienen roles y buscan soluciones a sus problemas comunitarios.

Las instituciones revelaron que las personas de la ruralidad, son más activas por la distancia que tienen con la capital comunal. Indican, que se preparan para recibir las precipitaciones debido a que pueden quedar aislados. A continuación, una tabla con capacidades y vulnerabilidades, emergentes del discurso.

\begin{tabular}{|c|c|c|}
\hline Subcategoría & Vulnerabilidades (-) & Capacidades (+) \\
\hline \multirow{4}{*}{ 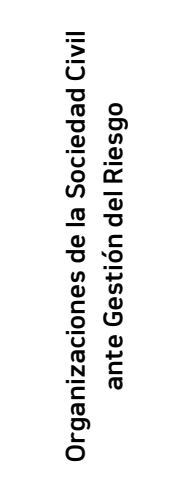 } & Área Rural & \\
\hline & $\begin{array}{l}\text { - Sin capacitaciones } \\
\text { - Falta socialización GRRD } \\
\text { - Planes sin participación }\end{array}$ & $\begin{array}{l}\text { - Organización comunitaria } \\
\text { - Comité de emergencia local } \\
\text { - Quieren ser parte del proceso de construcción } \\
\text { - Autovalentes } \\
\text { - Dirigentes orientan a vecinos }\end{array}$ \\
\hline & Área Urbana & \\
\hline & $\begin{array}{l}\text { - Sin preparación } \\
\text { - Falta socializar con la } \\
\text { comunidad } \\
\text { - Planes sin participación }\end{array}$ & $\begin{array}{l}\text { - Participan en mesa de trabajo } \\
\text { - Han socializado contradicciones ante instituciones } \\
\text { - Quieren ser parte del proceso de construcción }\end{array}$ \\
\hline
\end{tabular}

Tabla 17. Percepción sobre Organización de la Sociedad Civil ante Gestión del Riesgo, Capacidades y Vulnerabilidades Fuente: Autora, 2018

2. Subcategoría Instituciones para la Gestión del Riesgo: entendido desde estructura pública local, que puede prestar colaboración en etapas de GRRD. Es visualizado de diferentes maneras. Por un lado, en lo urbano indican que las instituciones no tienen un rol claro, que no planifican preventivamente, que tienen una respuesta inoportuna, sin obertura de espacios participativos de construcción. Por otro, en lo rural en la etapa de post emergencia, destaca comunicación directa con instituciones, para entregar catastros preliminares.

Además, los representantes institucionales se reúnen, proporcionando colaboración y fortaleciendo su respuesta. Indican que en instancias preventivas abren las puertas a la comunidad, pero en emergencias son herméticos para evitar rencillas. Se presenta una tabla que organiza capacidades y vulnerabilidades. 


\begin{tabular}{|c|c|c|}
\hline Subcategoría & Vulnerabilidades (-) & Capacidades (+) \\
\hline \multirow{4}{*}{ 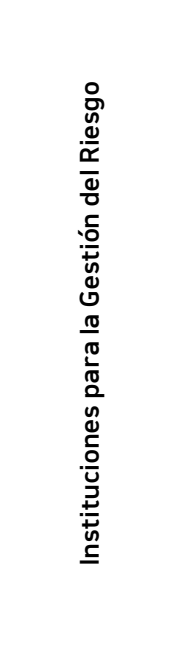 } & Área Rural & \\
\hline & $\begin{array}{l}\text { - Sin capacitación en GRRD } \\
\text { - Sin respuestas oportunas etapa pre } \\
\text { - Respuesta emergencista } \\
\text { - Sin espacios de participación vinculante }\end{array}$ & $\begin{array}{l}\text { - Dirigentes intermediadores entre comu- } \\
\text { nidad e instituciones } \\
\text { - Estructura y roles claros } \\
\text { - Aclaran tiempo de respuesta. }\end{array}$ \\
\hline & Área Urbana & \\
\hline & $\begin{array}{l}\text { - Sin socialización en GRRD } \\
\text { - Sin identificación de la persona } \\
\text { encargada } \\
\text { - Respuesta emergencista } \\
\text { - Sin espacios de participación vinculante } \\
\text { - Invisibles en el territorio } \\
\text { - Instituciones no preparadas }\end{array}$ & $\begin{array}{l}\text { - Reconocen la estructura } \\
\text { - Dirigentes orientan a sus vecinos }\end{array}$ \\
\hline
\end{tabular}

Tabla 18: Percepción sobre Instituciones ante Gestión del Riesgo, Capacidades y Vulnerabilidades Fuente: Autora, 2018

\section{CONCLUSIONES}

En este apartado se relevan aquellos aportes significativos de la investigación, en respuesta a la pregunta de investigación, relacionada a capacidades y vulnerabilidades que identifican las personas de Putre ante riesgos hidrometeorológicos derivados de lluvias estivales como amenaza.

Durante el año 2016, en localidades rurales y aisladas de Putre, Tignamar y Belén se permite reflexionar sobre problemas causados por lluvias estivales, bajo el Paradigma Social o de Vulnerabilidad en comprensión del riesgo. Este se basó en experiencias vividas y su relación con el entorno, para después entender la construcción social del riesgo que crean las personas.

El Análisis de Vulnerabilidades y Capacidades (AVC), permitió observar con mayor profundidad características del entorno social, político, económico y ambiental del territorio determinado. Los criterios del AVC, fueron organizados en 5 categorías de comprensión del entorno, permitiendo indagar en sus recursos, activos y medios, que desarrollan ante situaciones de riesgo.

Debido a las respuestas de los participantes, dentro de los resultados se hallaron diferencias entre áreas urbana y rural en la comuna de Putre, ya que los elementos identificados, están condicionados por la Estructura de Oportunidades (Pública, Sociedad civil y privada) presente en el territorio. Por ejemplo, al situarse ante Categoría Medios de Vida y disgregar en subcategorías, se observa una preparación comunitaria ancestral en la comuna de Putre, principalmente con la limpieza de canales de regadío, ligada a un trabajo comunitario que permite minimizar daños y continuar con el riego. Esta estructura social se construye y mantiene debido a las lluvias estivales, que son tratadas como un recurso escaso dentro de esta una región desértica. Además la periodicidad de la amenaza, provocó el desarrollo de instancias participativas, fortaleciendo vínculos y construyendo adaptación al medio habitado.

Del mismo modo, los testimonios dan cuenta del efecto que causa las ayudas estatales en el territorio, en especial en las relaciones comunitarias. Debido a que estos son otorgados individualmente, a la familia, por etnia o condición segregante o focalizante. Dicha situación ha provocado la separación de vínculos en las áreas urbanas. En cambio, ante la ausencia de tales condiciones ofrecidas por el Estado en el área rural, se observa unión en estas circunstancias.

Dentro del activo natural, las personas reconocen a las precipitaciones estivales como recurso de importancia en el desarrollo del poblado. Los putreños, indican que la condición climática, de aumento de frecuencia e intensidad, es observada como una amenaza, pues probablemente provoque riesgos de sequía o inundación, afectando tierras agrícolas y ganado, base del sustento familiar de los habitantes de Putre.

Los activos físicos son clasificados en 2 tipos, infraestructura comunitaria y familiar, destacando diferentes financiamientos y etapas para mejoras. En la primera financiando por el municipio se encuentra la construcción de sede. En cambio, con recursos propios se cambia techumbre. 
Los activos financieros, son observados como recursos para hacer frente a emergencia. Por este motivo se accede a recursos familiares, en caso de querer prevenir ante riesgos.

Además se percibe como vulnerabilidad la visión ante el ámbito educativo. Siendo la falta de capacitación y socialización situaciones negativas, en etapa de preparación en GRRD comunitaria. Con la finalidad de mejorar su actuar, ante otros tipos de amenazas.

Por lo demás, la influencia del área de salud, se explica en dos conceptos, uno es la relación institucional (tipo de atención al paciente) y la otra relacionada al estado de salud de las personas. En concordancia al vínculo institucional, existe un acercamiento de colaboración en lo rural, existiendo un paramédico con rol activo dentro de la organización local. En lo urbano, la relación existente es dentro de la formalidad de consejos de la sociedad civil.

La participación, es una instancia de desarrollo dentro del área rural, existiendo organización y adaptación para hacer frente a riesgos. En el área urbana se limita a la representación dirigencial ante asistencia. El ámbito trabajo es vulnerable para los medieros, los cuales no pueden recibir subsidios estatales, debido a que estos beneficios son para propietarios y no para arrendatarios de tierra. Esta situación es sentida por la comunidad, debido a que vecinos migrantes pierden cosechas, debido a su baja rentabilidad.

En criterio autoprotección, las personas indican una fuerte inyección de recursos estatales por PEDZE, que construye diferentes mejoras en las condiciones de habitabilidad comunitaria en áreas rurales aisladas. Sobre el concepto de fragilidad irrumpe el cambio climático como amenaza natural, como explicación en la disminución o aumento de las lluvias. Las personas enfatizan en su preparación y prevención ancestral que han tenido sobre técnicas de regadío. Asimismo, la resiliencia es vista como prácticas adaptativas en la cultura aymara, mediante acciones preventivas como limpieza y mantención de canales y techumbres.

La protección social se siente como oportunidades postemergencia. Clasificadas en relación a su origen (estatal, privado y familiar). En seguridad sobre medios de vida, existe un desconocimiento sobre planes de emergencia, por una baja participación y socialización. Se enfatiza la importancia de la sociedad civil en estos contextos, relacionado a participar en construcción de planes y divulgación de experiencias en GRRD (Complementar lo técnico y empírico), esperando una mejor actuación.

Dentro de gobernanza, la capacidad de conformación organizacional, se resuelve bien dentro de lo local. Debiéndose fortalecer para tender la Gestión del riesgo, crear vínculos institucionales y civiles, generar instancias participativas en construcción de planes y capacitaciones preventivas. Cambiar el actuar institucional de arriba hacia abajo.

Es importante resguardar experiencias de personas adultas, insumo para construir historias del habitar local, debido a que los sucesos pasados son parte de una cultura resiliente. Putre es afectada habitualmente por riesgos hidrometeorológicos como precipitaciones estivales, por tanto la comunidad ha adoptado prácticas preventivas y adaptativas.

Se enfatiza el aislamiento como principal vulnerabilidad en áreas rurales, debido a una baja conexión vial y comunicación, implicando afecciones en salud y producción. Esta situación fortalece prácticas ancestrales, manteniendo unidas familias y comunidades.

En relación a las categorías y subcategorías, su análisis muestra las dificultades que pueden presentar las comunidades al contar con una baja diversidad etaria, lo que es una vulnerabilidad al hacer frente a un riesgo, asimismo, la apropiación y conocimiento del territorio de los adultos mayores, proporciona una capacidad dentro de la historia del territorio, lo que permite conocer los impactos antes causados. Cabe destacar, que las comunidades deben disminuir o equilibrar las vulnerabilidades, mediante capacidades para hacer frente a los riesgos.

Se recomienda que el método AVC sobre construcción social del riesgo, se utilice como insumo empírico en políticas locales, por su importancia de comprensión y construcción del territorio por sus habitantes.

Asimismo, se debe complementar con información técnica, promoviendo adecuadas decisiones en la GRRD. También es importante que los encargados municipales puedan actualizar los análisis categoriales, trabajando en medidas preventorias y mitigadoras de vulnerabilidades. 
Además, fortalecer y crear capacidades, como medidas mitigadoras de riesgos. Incluso, ser potenciadas mediante un proyecto postulado al FNDR (Fondo Nacional de Desarrollo Regional), propuesta amparada en la comprensión dinámica de riesgos de desastres, protección de derechos y superación de la pobreza.

\section{REFERENCIAS}

Albornoz, C. \& Rodríguez, A. (2013a). Aplicación del modelo de presión del desastre: La eventual erupción del complejo volcánico Taapaca y la comunidad vecina de Putre, área altoandina del Norte de Chile. En 2do Seminario Internacional de Investigaciones sobre Vulnerabilidades de Desastres Socionaturales CIVDES. Santa Catarina, Brasil: Universidad Federal de Santa Catarina de Brasil.

Albornoz, C. \& Rodríguez, A. (2013b). Vulnerabilidad demográfica y exposición de la población de Putre ante la eventual erupción del complejo volcánico Taapaca. En S. C. Geográficas, Anales Sociedad Chilena de Ciencias Geográficas. Santiago de Chile: Sociedad Chilena de Ciencias Geográficas (pp. 19-25).

Arica Mía. (Agosto, 2016). Con participación aymara la región aporta a la Estrategia sobre cambio climático y recursos vegetacionales. Disponible en: http://www.aricamia.cl/con-participacion-aymara-la-region-aporta-a-la-estrategia-cambio-climatico-y-recursos-vegetacionales/ [visitado el 20 de Julio 2018]

Blaikie, P., Cannon, T., Davis, I. \& Wisner, B. (1996). Vulnerabilidad: El entorno social, político y economico de los desastres. Perú: LA RED.

Bravo Flores, S. (2002). Invierno del Altiplano, implicancias desfavorables y favorables en el desarrollo de la $1^{\circ}$ y $2^{\circ}$ región de Chile. En Congressos Brasileiros de Meteorologia 2ooo. Foz de Iguazu: ANAIS (pp. 1156-1162).

CARITAS Chile. (2015). Prácticas de Gestión Local del Riesgo de Desastre: Hacia la co-construcción de comunidades resilientes. Santiago: Caritas Chile.

Radio Cooperativa. (Mayo, 2016). Fuertes lluvias cortaron caminos secundarios en Arica y Parinacota. Disponible en: http://www.cooperativa.cl/noticias/pais/tiempo/fuertes-lluvias-cortaron-caminos-secundarios-en-arica-y-parinacota/2016-02-20/102452.html [visitado el 20 de Julio 2018]

Dirección General de Programación Multianual del Ministerio de Economía y Finanzas DGPM-MEF. (2007). Conceptos asociados a la gestión del riesgo de desastres en la planifica-ción e inversión para el desarrollo. Documento 1, Serie Sistema Nacional de Inversión Pública y la Gestión del Riesgo de Desastres. Lima: DGPM, MEF \& PDRS-GIZ.

EIRD. (2016). Estrategia Internacional para la Reducción de Desastres. Disponible en: http://www.eird. org/esp/terminologia-esp.htm [visitado el 20 de Julio 2018]

República de Chile. (1980). Constitución Política de la República de Chile. Decreto Supremo $N^{\circ} 100$. Santiago de Chile: Gobierno de Chile.

Federación Internacional de Sociedades de la Cruz Roja y de la Media Luna Roja FICR. (20o6). ¿Qué es el AVC? Introducción al Análisis de Vulnerabilidades y Capacidades. Ginebra: Publicaciones FICR.

Flick, U. (2007). Introducción a la investigación Cualitativa. Madrid: Ediciones Morata.

Fundación Superación de la Pobreza. (2016). Voces desde las pequeñas localidades de Arica y Parinacota: Entre la agonía y la oportunidad de renacer. Arica, Chile: FSP.

Fundación Superación de la Pobreza. (2013). Voces de la Pobreza, significados, representaciones y sentir de personas en situación de pobreza a lo largo de Chile. Santiago de Chile: FSP

García Acosta, V. (2005). El riesgo como construcción social y la construcción social de Riesgos. Desacatos, 19, 11-24.

Gobierno Regional de Aysén. (2017). Plan de desarrollo para Zonas extremas de Aysén (PEZDE). Aysén, Chile: GORE Aysén. Disponible en: http://www.goreaysen.cl/controls/neochannels/neo_ch237/neochn237.aspx [visitado el 20 de Julio 2018]

Ilustre Municipalidad de Putre. (2014). Plan de Desarrollo Comunal Putre. Putre: IG Pronorte.

Instituto Nacional de Estadísticas INE. (2017). Censo 2017. Instituto Nacional de Estadísticas Arica y Parinacota. Disponible en: http://ine-chile.maps.arcgis.com/apps/webappviewer/index.html?i$\mathrm{d}=69596 \mathrm{c} 770 \mathrm{c} 714200 a 7 \mathrm{bd} 423 \mathrm{f} 40 \mathrm{e}$ b46c [visitado el 20 de Julio 2018] 
Kaztman, R. \& Filgueira, C. (1999). Marco conceptual sobre activos, vulnerabilidades y estructura de oportunidades. En Kaztman, R. \& Filgueira, C. (Eds.), Activos y estructuras de oportunidades. Estudios sobre las raíces de la vulnerabilidad social en Uruguay. Montevideo: CEPAL (pp. 16-36).

Lavell, A. (2000). Desastres y Desarrollo: Hacia un Entendimiento de las Formas de Construcción Social de un Desastre: El Caso del Huracán Mitch en Centroamérica. En Garita, N. \& Nowalski, J. (Eds.), Del Desastre al Desarrollo Sostenible: El Caso de Mitch en Centroamérica. San José: BID (pp. 8-45).

Lavell, A. (2003). La gestión local del riesgo. Nociones y precisiones entorno al concepto y la práctica. Ciudad de Guatemala: PNUD.

Maskrey, A. (1989). El manejo popular de los Desastres Naturales Estudios de Vulnerabilidad y Mitigación. Lima: Tecnología Intermedia (ITDG).

Max-Neef, M. (1998). Desarrollo a Escala Humana, Conceptos, aplicaciones y algunas reflexiones. Barelona: Icaria.

Mayan, M. (2001). Una Introducción a los Métodos Cualitativos: Módulo de Entrenamiento para Estudiantes y Profesionales. Iztapalapa, México: Qual Institute Press.

Ministerio de Desarrollo Social de Chile MDS. (2013). Presentación resultados Encuesta Casen 2013. Santiago de Chile: MSD. Disponible en: http://observatorio.ministeriodesarrollosocial.gob.cl/ casen-multidimensional/casen/casen_2013.php [visitado el 20 de Julio 2018] 